the second phase of revision, linearizations are being developed, with highest priority being given to the Joint Linearization for Mortality and Morbidity Statistics (JLMMS). A special linearization based on ICECI is envisaged.

Conclusions The form and content of the JLMMS are still in flux, but are expected to be in largely final draft by mid-2016. Injury researchers need to be aware of the forthcoming changes to the ICD, which will impact on injury statistics worldwide.

\section{TRAUMA CARE EVALUATION}

1,2Belinda Gabbe. ' Monash University, Australia; ${ }^{2}$ Swansea University, UK

10.1136/injuryprev-2016-042156.45

Background While the global burden continues to rise, injuryrelated mortality rates have declined in many high-income countries. A key contributor to reduced mortality has been implementation of trauma systems with improved care of injured patients substantially enhancing the likelihood of surviving serious injury. The focus has now shifted to improving the quality of survival and reducing the burden of non-fatal injury. However, there is limited understanding of how well patients recover, how long this takes, and the proportion of the injured population who go on to experience lifelong disability.

Methods The state of Victoria, Australia, using the populationbased Victorian State Trauma Registry as a data spine, is using coordinated data linkage, longitudinal qualitative studies, and routine long-term patient follow-up to evaluate trauma care through acute care, rehabilitation and community reintegration.

Results Key findings include: i) demonstrated reduced burden, measured in Disability Adjusted Life Years, of road traffic injury following implementation of an organised trauma system; ii) improved mortality and functional outcomes for patients following redesign of the state's retrieval system; iii) improvement in patient-reported outcomes to 2-years post-injury but variable recovery trajectories for key patient groups; and iv) profound issues with discharge planning, and post-discharge care coordination of trauma patients.

Conclusions Clinical data represents only a component of what we need to know to understand the impact of clinical practice and healthcare policy in trauma. Insight into patient experiences and pathways, healthcare and disability service needs, and factors that facilitate and impede recovery are needed to improve trauma system design and better meet the needs of injured patients.

\section{THE NATIONAL EMERGENCY MEDICAL SERVICES INFORMATION SYSTEM (NEMSIS): PREHOSPITAL CARE DATA ON INJURY}

${ }^{1}$ Holly Hedegaard, ${ }^{2} \mathrm{~N}$ Clay Mann. ${ }^{1}$ Centers for Disease Control and Prevention National Centrefor Health Statistics, USA; ${ }^{2}$ University of Utah, USA

\subsection{6/injuryprev-2016-042156.46}

Background The National Emergency Medical Services Information System (NEMSIS) is the repository for data on emergency medical services (EMS) in the United States (US). Standard data elements are collected by local EMS providers, then aggregated at the state level and submitted to the national database. Data from NEMSIS are used to evaluate care delivery, compare regional differences, inform EMS provider training and generate research hypotheses.
Methods Data were accessed using the NEMSIS on-line data cube. Nearly 25.5 million patient care reports were submitted in 2014. The subset of injury events were selected using Type of service $=911$ response and Possible injury $=$ Yes.

Results More than 3.3 million records for possible injury events were reported in 2014, with 50\% involving females and with $13 \%$ involving patients aged $0-19,50 \%$ aged $20-59$ and $36 \%$ aged $60+$. Cause of injury was reported for $72 \%$. Of events with a known cause, falls accounted for $46 \%$, land transport 33\%, struck by blunt object $11 \%$ and drug poisoning $4 \%$. Age and sex distribution varied by cause of injury. Treatment was provided in $85 \%$ of events. Procedures were reported as being performed on $60 \%$ of patients. Common procedures included venous access (33\%), spinal immobilisation (28\%), cardiac monitoring (21\%), pulse oximetry (20\%), wound care $(14 \%)$ and splinting $(6 \%)$. Medications were reported as being given to $19 \%$ of patients. For those receiving medications, the most common medications were fentanyl (17\%), morphine (10\%), ondansetron $(10 \%)$ and naloxone $(2 \%)$. Nearly $90 \%$ of the treated patients were transported from the scene to a facility.

Conclusions Analysis of NEMSIS data provides insight into the provision of EMS care to injured patients in the US. This data system may serve as a model for other countries in how to capture standard information from prehospital providers. Continued efforts to improve completeness of reporting will enhance the utility of these data for injury research.

\section{DISABILITY WEIGHTS FROM THE INJURY VIBES COLLABORATION}

\begin{abstract}
1,2Ronan A Lyons, ${ }^{2}$ Belinda J Gabbe, ${ }^{2}$ Pam M Simpson, ${ }^{3}$ James E Harrison, ${ }^{4}$ Shanthi Ameratunga, ${ }^{5}$ Sarah Derrett, ${ }^{6}$ Suzanne Polinder, ${ }^{7}$ Frederick P Rivara. ${ }^{1}$ Farr Institute, Swansea University Medical School, UK; ${ }^{2}$ Department of Epidemiology and Preventive Medicine, Monash University, Melbourne, Australia; ${ }^{3}$ Research Centre for Injury Studies, Flinders University, Adelaide, South Australia; ${ }^{4}$ Section of Epidemiology and Biostatistics, School of Population Health, University of Auckland, Auckland, New Zealand; ${ }^{5}$ Injury Prevention Research Unit, Department of Preventive and Social Medicine, Dunedin School of Medicine, University of Otago, Dunedin, New Zealand; ${ }^{6}$ Department of Public Health, Erasmus MC, Rotterdam, The Netherlands; 'Departments of Paediatrics and Epidemiology, University of Washington, Seattle, USA
\end{abstract}

\subsection{6/injuryprev-2016-042156.47}

Background Disability weights (DWs) are an integral part of deriving the years lived with disability (YLD) component of disability adjusted life years (DALYs). DWs can be derived through different methods including panels comprising of experts, the general population judging the impact of conditions on loss of health, or through follow-up data on the health-related quality of life of groups of injured patients. The Validating and Improving injury Burden Estimates Study (Injury-VIBES) sought to create new injury DWs by combining data from six of the largest injury outcome studies, that were conducted in Australia, New Zealand, United Kingdom, Netherlands and USA.

Methods Data were combined in an individual level meta-analysis from the Victorian State Trauma Registry, Victorian Orthopaedic Trauma Outcomes Registry, Dutch Injury Patient Survey, UK Burden of Injury Study, Prospective Outcomes of Injury Study, and the National Study on Costs and Outcomes of Trauma. DWs were calculated for individual and grouped ICD10 diagnosis codes and established nature of injury classifications. Twelve month annualised DWs (assumed to be indicative of permanent health loss) were calculated separately for cases discharged 